\title{
A Numerical Method for Pricing Discrete Double Barrier Option by Lagrange Interpolation on Jacobi Nodes
}

\author{
Amirhossein Sobhani ${ }^{1}$ and mariyan milev ${ }^{2}$ \\ ${ }^{1}$ Semnan University \\ ${ }^{2}$ University of Food Technology-Plovdiv, Head of Mathematics and Physics Bulgaria, \\ Plovdiv.
}

June 14, 2021

\begin{abstract}
In this paper, a rapid and high accurate numerical method for pricing discrete single and double barrier knock-out call options is presented. With regard to the well-known Black-Scholes model, the price of an option in each monitoring date could be calculated by computing a recursive integral formula that is based on the heat equation solution. We have approximated these recursive solutions with the aid of Lagrange interpolation on Jacobi polynomial nodes. After that, an operational matrix, that makes our computation significantly fast, has been derived. In some theorems, the convergence of the presented method has been shown and the rate of convergence has been derived. The most important benefit of this method is that its complexity is very low and does not depend on the number of monitoring dates. The numerical results confirm the accuracy and efficiency of the presented numerical algorithm.
\end{abstract}

\section{Hosted file}

option pricing Jacobi.pdf available at https://authorea.com/users/419703/articles/526112-anumerical-method-for-pricing-discrete-double-barrier-option-by-lagrange-interpolationon-jacobi-nodes 